\title{
Back on track - On the role of the microtubule for kinesin motility and cellular function
}

\author{
STEFAN LAKÄMPER ${ }^{1}$ and EDGAR MEYHÖFER ${ }^{2, *}$ \\ ${ }^{1}$ Physics of Complex Systems, Vrije Universiteit Amsterdam, De Boelelaan 1081, 1081 HV, Amsterdam, The \\ Netherlands; ${ }^{2}$ Department of Mechanical Engineering, University of Michigan, 2350 Hayward St., Ann Arbor, MI, \\ 48109, USA
}

Received 18 July 2005; accepted in revised form 8 December 2005

\begin{abstract}
The evolution of cytoskeletal filaments (actin- and intermediate-filaments, and the microtubules) and their associated motor- and non-motor-proteins has enabled the eukaryotic cell to achieve complex organizational and structural tasks. This ability to control cellular transport processes and structures allowed for the development of such complex cellular organelles like cilia or flagella in single-cell organisms and made possible the development and differentiation of multi-cellular organisms with highly specialized, polarized cells. Also, the faithful segregation of large amounts of genetic information during cell division relies crucially on the reorganization and control of the cytoskeleton, making the cytoskeleton a key prerequisite for the development of highly complex genomes. Therefore, it is not surprising that the eukaryotic cell continuously invests considerable resources in the establishment, maintenance, modification and rearrangement of the cytoskeletal filaments and the regulation of its interaction with accessory proteins. Here we review the literature on the interaction between microtubules and motor-proteins of the kinesin-family. Our particular interest is the role of the microtubule in the regulation of kinesin motility and cellular function. After an introduction of the kinesin-microtubule interaction we focus on two interrelated aspects: (1) the active allosteric participation of the microtubule during the interaction with kinesins in general and (2) the possible regulatory role of post-translational modifications of the microtubule in the kinesin-microtubule interaction.
\end{abstract}

\section{Kinesin-microtubule interaction}

Kinesins are a large and diverse family of microtubule based motors (Vale, 2003; Lawrence et al., 2004). They all share a high degree of sequence homology in the motor domain with the founding member, conventional kinesin (Kinesin-1) (Brady, 1985; Vale et al., 1985). Conventional kinesins are $(+)$-end directed motors that are hetero-tetramers of two heavy and two light chains (Kuznetsov et al., 1988). The N-terminal motor domains of Kinesin-I contain both the ATPase and MT-binding activity (Kuznetsov et al., 1989; Scholey et al., 1989). They are flexibly linked to the $\alpha$-helical neck via the so-called neck-linker, which is believed to confer important conformational changes during the generation of movement (Rice et al., 1999). Coiled-coil interactions between the neck domains of two heavy chains lead to dimerization of Kinesin-1 (de Cuevas et al., 1992). An extended coiled-coil of about $60 \mathrm{~nm}$ forms the stalk, which is interrupted by flexible hinges and kinks (Kirchner et al., 1999). A small globular, C-terminal tail is responsible for light chain (KLC)

* To whom correspondence should be addressed. Phone: + 1-734 647-7856; Fax: +1-734-615-6647; E-mail: meyhofer@umich.edu binding (Johnson et al., 1990; Verhey et al., 1998). Tail and KLC are important for both the binding of various cargo adapters and regulation of the unbound motor through inhibitory back-folding (Goldstein, 1993; Verhey et al., 1998; Coy et al., 1999; Seiler et al., 2000; Goldstein, 2001).

Kinesin-I has the remarkable ability to coordinate the ATPase activity of its two heads such that a single molecule is capable of moving processively along the microtubule for micrometer distances (Howard et al., 1989; Hackney, 1994; Vale et al., 1996; Gilbert et al., 1998; Lakamper et al., 2003). By alternately advancing the motor domains along the microtubule in a handover-hand fashion Kinesin-1 can take hundreds of $8 \mathrm{~nm}$ steps and produce stalling forces of up to 5-7 pN (Svoboda et al., 1993; Meyhofer and Howard, 1995; Asbury, 2003; Yildiz et al., 2004; Carter and Cross, 2005). Processivity is enabled by strictly asynchronous, ATP-driven cycling between weak and strong binding states of each of the two heads. While ATP-bound and nucleotide-free motor-domains are strongly bound to the microtubule, ADP-bound heads are weakly bound. A working hypothesis for this mechano-chemical cycle is summarized in Figure 1. In solution, both heads of dimeric kinesin are in the 


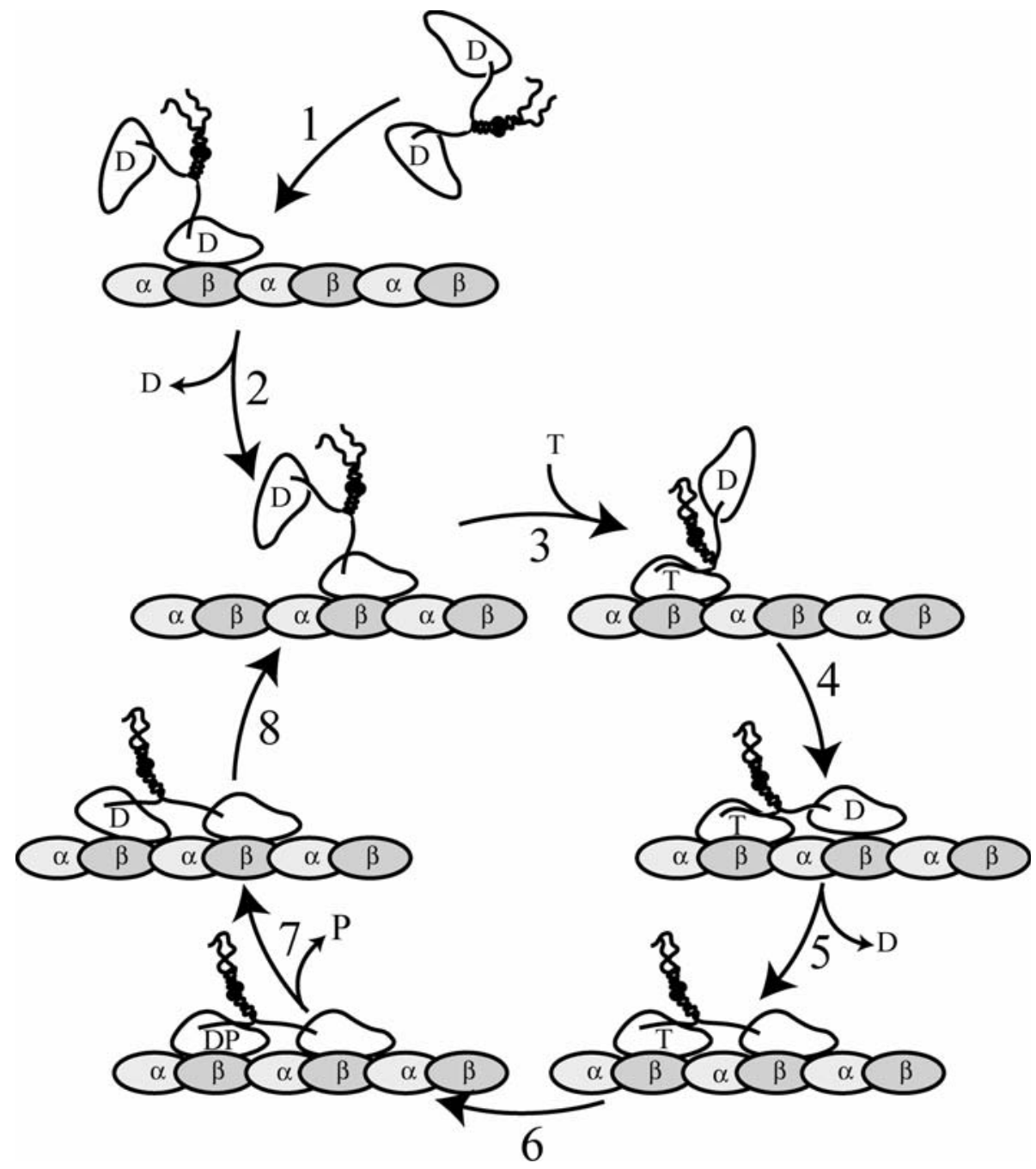

Fig. 1. Working model for the processive movement of Kinesin-I. Upon collision with the microtubule (1), the dimeric motor quickly looses the ADP molecule from the binding head (2), spatial constraints require binding of ATP and reorientation of the neck-linker (3) before the second head can bind to the next available binding site (4) and release ADP (5) to generate an internally strained doubly bound motor. It has been shown that the rearward strain on the leading head prevents ATP-binding, and we hypothesize that the forward strain on the rear head accelerates ATP-hydrolysis (6), which leads to rapid phosphate release (7) and dissociation of the ADP-kinesin-head (8) which allows the cycle to start again. Based on Lakamper and Meyhofer (2005).

ADP-state. Upon (productive) collision with the microtubule, binding triggers rapid ADP-release of one head, leading to the strongly bound nucleotidefree state, while the second head is unable to bind and thus retains its ADP. Only the rearrangement of the neck-linker upon ATP-binding on the so-called 'rear head' allows the second head to bind and to loose its ADP. Binding of the second head is believed to introduce intra-molecular strain, (i.e. backwards in the front head and forward in the rear head) and to (a) prevent ATP-binding to the front head in order to keep this head in the strongly bound state and (b) trigger ATP-hydrolysis and phosphate-release in the rear head. This latter process generates a weakly bound ADP-head which can subsequently dissociate and reorient such that the nucleotide-free head (now rear) can bind ATP and start the cycle again. The most prominent conformational changes accompanying nucleotide-changes in the kinesin head are (a) the immobilization of the otherwise highly mobile necklinker upon ATP-binding (Rice et al., 1999) and (b) a rotation of the kinesin core associated with nucleotidehydrolysis (Vita et al., 2004). In the latter conformatonal change the kinesin motor-domain rotates about $20^{\circ}$ counter-clockwise while increasing the affinity to the microtubule binding-interface. In order to understand how the structural rearrangements in the motor-domain drive kinesin's motility knowledge of the location and nature of the interaction-sites of kine$\sin$ and the microtubule is of considerable importance.

Interaction sites between kinesin and ITs

Where and how do the kinesin motors and ATs interact? The obvious interaction site is the classical nucleotide-dependent binding site (NDBS). In order to adapt to various cellular functions, kinesins not only 
developed variations in the number, arrangement and function of classical binding sites (N-terminal and C-terminal motors, internal motors, as well as monomeric, homo- and hetero-dimeric and tetrameric motors), but also developed additional, nucleotideindependent MT-binding sites (NIBS) which aid in and/or modulate the interaction of the predominantly conserved kinesin-MT interaction site or serve other functions in the interaction with the microtubule. While the functional and structural distinction is usually rather clear, the influence of the NIBS can be so intimately intertwined in the mechanism of the NDBS that a distinction becomes less transparent. Interestingly, both NIBS and NDBS have a rather limited binding surface on the outer rim (Wendt et al., 2003) of the MT and might therefore compete with and influence each other or other microtubule associated proteins, like tau (Bonnet et al., 2001; Seitz et al., 2002). Of these NDBS and NIBS, the classical interaction between kinesin and the MT is best understood structurally.

\section{$N D B S$}

To identify residues of kinesin interacting with the tubulin-surface and vice versa several approaches have been used. EM-studies first established the interaction sites and showed that the binding sites for all kinesins examined so far (processive, non-processive, C-terminal, internal and N-terminal motors) appear to be very similar and primarily differ structurally in the more distal parts of the motor (see for example Hirose et al., 1995; Hoenger et al., 1995; Hoenger and Milligan, 1997; Hirose et al., 1999 and references cited therein). This work has been complemented by sophisticated biochemical and genetic approaches. Recently, for example, elegant combinations of cross-linking or foot-printing experiments with mass-spectroscopy or Western-blot analysis confirmed that the motor-domains of the kinesins studied so far share very similar interaction sites (Alonso et al., 1998). The MT binding site of conventional kinesin has been mapped in detail bio-chemically using cross-linking studies and alaninescanning techniques (Song and Mandelkow, 1993; Tucker and Goldstein, 1997; Woehlke et al., 1997). Docking of the crystal-structure into 3D-electron-density maps of decorated microtubules confirmed the biochemical findings (Hoenger et al., 1995). The switch II cluster $(\alpha 4 / \mathrm{L} 11 / \alpha 5)$ of conventional kinesin interacts with the helix $\mathrm{H} 12$ of $\beta$-tubulin while the region $\beta 5 \mathrm{a} / \mathrm{b}$ forms partial contacts with both the $\beta$ - and $\alpha$-subunit (Song et al., 2001; Woehlke, 2001). Alanine-scanning approaches allowed determining the contribution of individual, surface-exposed kinesin-residues to the binding affinities. Interestingly, the most important residues are predominantly charged residues. Here substitution of negatively charged residues led to an increase in affinity (reduced $\mathrm{K}_{\mathrm{M}} \mathrm{MT}$ ) while a substitution of positively charged residues found in close proximity led to a decrease in affinity (increased $\mathrm{K}_{\mathrm{M}} \mathrm{MT}$ ). Interestingly, these residues are conserved (in both the superfamily and the Kinesin-I family) and cluster in two regions, loop 7 and loop 8 (loop 7 being in close proximity to the switch I region) and L11/ $\alpha 4$ (switch II-loop) together with L12/ $\alpha 5$. While it is not completely understood if some of the substitutions are influencing affinity directly or indirectly, this study clearly highlights the importance of the structural elements and the predominantly electrostatic nature of the interaction (Figure 2). The identification of these regions is in agreement with findings from Nitta et al. who found crystallographic evidence that the helix $\alpha 4$,
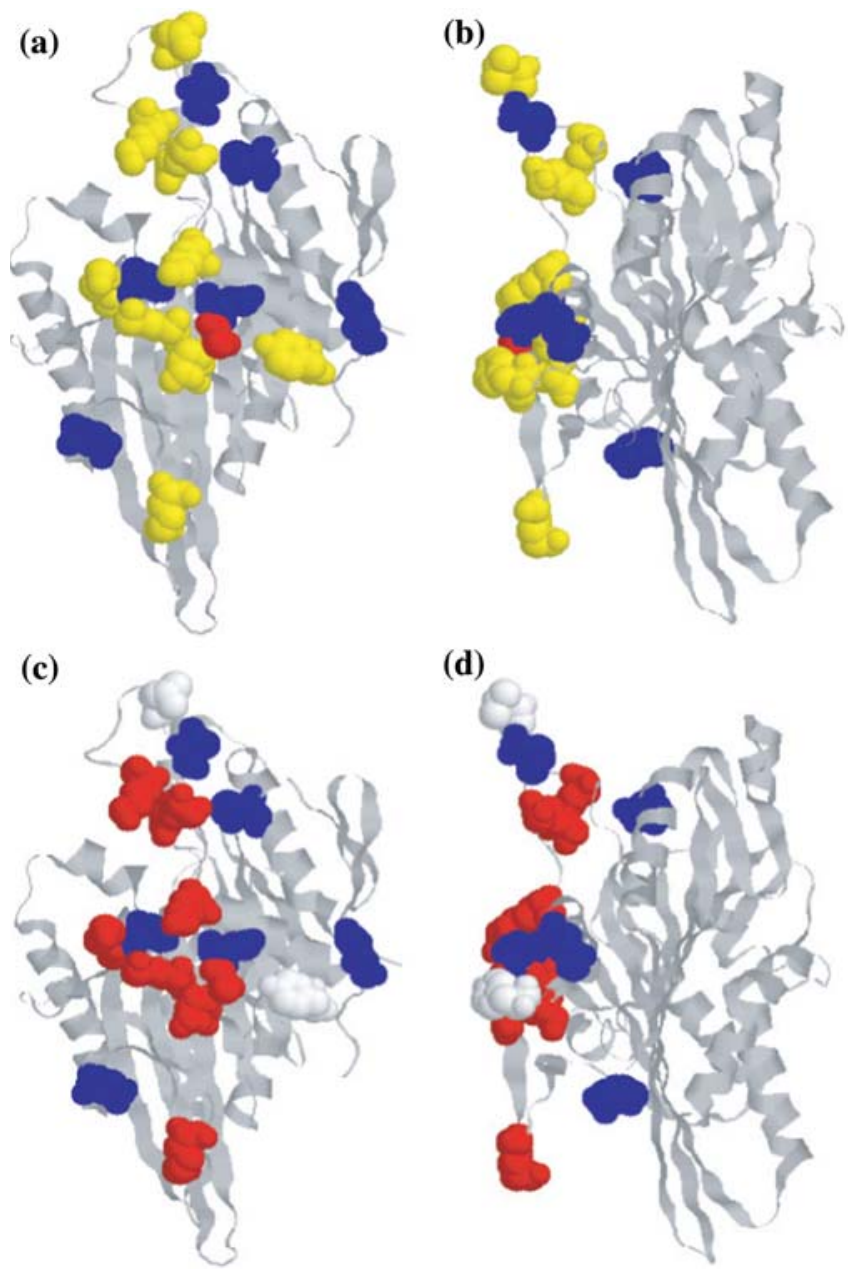

(d)

Fig. 2. The microtubule-binding site of Kinesin-I. (a) and (c) show the kinesin motor-domain after Kull (1996) with the nucleotide-binding site located on the back. (b) and (d) show a side-view of the molecule with the microtubule-binding side facing left. The microtubule plusend would be oriented downwards. (a) and (b) replacement of residues with alanine result in affinity decreases (yellow) and increases (blue) in some residues. In the side-view it can be seen how these residues cluster on the microtubule-binding surface. (c) and (d) representation of the residues according to their charge. Residue L248 (top) and Tyr274 (middle) are in light gray, while positively charged residues are colored in red and negatively charged residues are blue. In the comparison between panels $\mathrm{a} / \mathrm{b}$ and $\mathrm{c} / \mathrm{d}$ it can be seen that replacement of positively charged residues leads to affinity decrease while replacement of negatively charged residues leads to an increased affinity. 
which connects the MT-interacting loop11 and 12 and is nested between the $\alpha$ - and $\beta$-subunits of tubulin, tilts significantly upon ATP-binding (Nitta et al., 2004).

While the conformational changes in the kinesin motor domain upon MT-binding have received considerable attention there is comparatively little known about the active participation of the MT in kinesinmotility. It could be shown that MTs grown in the presence of the non-hydrolysable GTP-analog GMPCPP are transported $30 \%$ faster by conventional kinesins (Vale et al., 1994). They were also less often running in curved paths than GDP-MTs. In agreement with the structural interpretation that GTP-protofilaments adopt a less curved conformations, they were shown to be less curved and more rigid. Interestingly, the in-solution ATPase-activity of kinesin on GMPCPP MTs was unaffected (Vale et al., 1994). However, this result suggested that the tubulin within MTs might be able to adopt different conformations and that either the rigidity or the actual tubulin-conformation might influence kinesin motility. The fact that the microtubule can adopt a distinct conformation which alters the motile behavior of Kinesin-I can be seen as an initial indication that binding of the motor to the microtubule might not only trigger conformational changes in the motor (which for example lead to ADP-release) but also that possibly subsequent rearrangements in the kinesin-motor domain might force the microtubule-structure to rearrange.

In fact, changes in the tubulin structure upon kinesin binding were reported in cryo-EM-studies of ncddecorated MTs (Hoenger and Milligan, 1997). But only recent, refined cryo-EM-studies with 15-mer MTs decorated with monomeric constructs of the fast fungal kinesin NcKin could reveal more details. 15-mer MTs, which display a right-handed super-twist, show distinct differences in electron-density-maps with and without decoration with motors (Krebs et al., 2004). Most prominently, binding of the motor to the $\beta$-subunit leads to continuous core electron-density along the protofilaments which is mainly brought about by structural changes in the $\alpha$-subunit. Together with the observation that single, stabilized protofilaments can only be found in preparations that were incubated with motor molecules but never in plain MT samples, these findings are interpreted as a clear indication of axial stabilization of the protofilament. Concomitantly, a slight reduction of density on the outer rim of tubulin was observed, corresponding to the motor binding site. The stabilization of axial protofilaments is reminiscent of the increased gliding speed observed with GMP-CPP-MTs, (Vale et al., 1994). While it is unclear which mechanism is responsible for the increased speeds of GMP-CPP-MTs (the ATPase activity is apparently unchanged) and how the above structural and functional observations relate in detail, these observations argue for allosteric effects associated with kinesin binding, perhaps even cooperative axial effects over several tubulin subunits as inferred form the
GTP-cap-hypothesis. Interestingly, a recent report provides some evidence in support of long-range cooperative binding of kinesin motors to microtubules, binding of kinesin coated beads to a MT led to increased binding frequency in the vicinity of the initial bead (Muto et al., 2005). This effect was only observed in the presence of ATP, but not AMP-PNP and ADP. Control experiments with hetero-dimeric motors consisting of a wt-kinesin and a hydrolysis-incompetent mutant (E236A) confirmed that ATP-hydrolysis is essential for the observed cooperative binding. It could further be shown that the cooperativity is slightly biased towards the $(+)$-end of the MT. While the data suggest cooperative effects, the idea of cooperative binding effects over a distance of 10-50 tubulin subunits is challenging. It remains to be determined how much energy is required and/or which conformational changes are necessary in the MT-lattice and the tubulin-monomers to allow for an increased binding rate.

\section{NIBS}

NIBS have first been described for the tail of the (-)-end directed and non-processive kinesin ncd (Karabay and Walker, 1999a,b, 2003). The N-terminal position of the NIBS of ncd is reminiscent of a conventional kinesin cargo-binding domain and has binding properties that are similar to microtubule associated proteins (MAPs, Wendt, 2003), suggesting that the MT-crosslinking effects reported in vitro reflects important functions of this mitotic motor in vivo, namely in the mitotic spindle (Karabay and Walker, 1999b). Other NIBS are located in the vicinity of the motor domain and have been shown to increase the affinity of the motor, but not the catalytic turnover. These affinity-increasing NIBS have been identified in $\mathrm{N}$-terminal extensions of the BimC motor domain (Stock et al., 2003), but also in internal loops within the motor domain, the so called K-loop of KiflA of the Unc104-family (Okada and Hirokawa, 2000). The K1A K-loop increases the affinity to the native MT so drastically, that monomeric Kif1A motors could be observed to diffuse in one dimension along the MT in a pseudo-processive manner (Okada and Hirokawa, 1999). A comparable mechanism has been suggested for the MT-depolymerizing kinesin MCAK (Ovechkina et al., 2002) while a similar mechanism based on electrostatic interactions of the dimeric neck of conventional kinesins with the microtubule (Thorn et al., 2000; Wang and Sheetz, 2000), has recently been challenged by others (Skiniotis et al., 2004) and us (Lakamper and Meyhofer, 2005, discussed in detail below). Attractive interactions of the stalk with the MT, however, have convincingly been shown to increase the affinity of the motor HsKID (Shiroguchi et al., 2003). Combinations of multiplied classical binding sites and NIBS can for example be found in the tetrameric motor BimC (Kashina et al., 1996; Shiroguchi et al., 2003). 
There is little information about the actual structure and MT-interaction sites of NIBS located close to the motor domain. However, they all seem to share a predominantly electrostatic character. More information about these structures and their interaction with the MT might be inferred from the NIBS of the ncd-tail. Actually, the ncd-tail domain contains two NIBS, a weaker binding site at position 115-187 and a stronger (N-terminal residues 83-100, (Karabay and Walker, 1999a). Based on cryo-EM studies the ncd-fragment NT6, which contains both binding regions, displays no prominent globular domain and behaves rather like the filamentous MAP tau. However, the studies with NT6 revealed four sites of increased electron density on the outer rim of the microtubule (Wendt et al., 2003).

Interestingly, the two MT binding sites of the tail of ncd interact with two regions of the most C-terminal structures of both the $\alpha$ - and $\beta$-subunit of tubulin: (1) the H12 on $\alpha$-tubulin (ordered), (2) the $\mathrm{C}$-terminus on $\alpha$-tubulin (disordered, $\alpha$-E-hook), (3) the C-terminal $\mathrm{H} 12$ on $\beta$-tubulin (ordered) and (4) the $\mathrm{C}$-terminus on $\beta$-tubulin (disordered, $\beta$-E-hook (Karabay and Walker, 2003). Sites 1 and 3 together correspond to the classical binding site of conventional kinesin (Wendt, 2003). The transition H11-H12 loop and the H12-helices contain a significant number of negatively charged residues ( $\mathrm{E}$, acidic cluster), suggesting that the tail interactions are very much mediated by electrostatic interactions, which is in agreement with its MAP-like structural appearance and the reported salt sensitivity of NT6-interactions (Karabay and Walker, 2003). Interestingly, the finding of a MAP-like protein binding in this region suggests competition for binding of motors which has been described for conventional kinesin and MAPs by Seitz (2002). The other two other binding sites, 2 and 4 , correspond to the estimated position of the so called E-hooks of tubulin (Karabay and Walker, 2003). These 12-18 AA long domains are not resolved in the crystal structures and are therefore interpreted to be highly flexible (Nogales et al., 1998). The E-hooks show, however, a slight tendency to form helical structures in vitro (Jimenez et al., 1999). The E-hooks can be removed from tubulin subunits by proteolytic treatment with subtilisin (Bhattacharyya et al., 1985; Sackett et al., 1985). The removal of the $\beta$-E-hook reduces not only the binding stochiometry from 4 to 3 , but also drastically reduces the overall affinity of NT6 to the microtubule. Further removal of the $\alpha$-E-hook leads to a binding stochiometry of two and lower affinities. The remaining two NT6 bind to the acidic cluster in the ordered H12. Furthermore, it could be shown that the interaction between the MT and NT6 is highly salt-sensitive (Karabay and Walker, 2003). These findings of NT6interactions with the E-hook provide a structural basis for the electrostatic interactions of other NIBS and pinpoint the importance of the E-hook.

\section{Specific roles for the E-hook in microtubule-Kinesin-I} interactions

The E-hook has mainly been described in the context of NIBS-mediated binding. For example, the pseudoprocessive, one dimensional diffusion of monomeric Kif1A constructs on native microtubules (Okada and Hirokawa, 1999) is abolished by either removing the highly positively charged K-loop-insertion in the motor or by removal of the E-hook (Nitta et al., 2004; Okada and Hirokawa, 2000). This and the high salt-sensitivity suggest strong electrostatic interactions, similar to what has been described for the ncd-tail. Equivalent experimental approaches have been performed to asses the role of the E-hook for KID (Shiroguchi et al., 2003), MCAK (Ovechkina et al., 2002), BimC (Stock et al., 2003) and conventional kinesin. While the results for KIF, KID, MCAK and BimC suggested that the main role of the E-hook is an increase in the kinesin-MT affinity (in agreement with electrostatic interactions), the removal of the E-hook led to surprisingly different effects in conventional kinesins, namely a reduction in speed and processivity (Wang and Sheetz, 2000). On the basis of elegant experimental approaches, an electrostatic interaction of the E-hook with the dimeric neck of conventional kinesin was proposed to be the structural basis for the observed E-hook function. (Thorn et al., 2000).

In contrast to this interpretation, it has been shown in earlier experiments that both E-hooks efficiently cross-link directly to the kinesin motor domains (Tucker and Goldstein, 1997), although there is a preference for the $\beta$-subunit as demonstrated in another study (Song and Mandelkow, 1993). The cross-links to the motor-domain are very likely to attach to regions that correspond well with the interaction sites obtained by alanine scanning methods of kinesin (Woehlke et al., 1997). Removal of the E-hooks and addition of E-hook peptides reduced cross-linking efficiency (Tucker and Goldstein, 1997). Two more recent lines of evidence further support the hypothesis that the E-hook is not just passively associating with NIBSs of the motor, but directly interacting with the core motor domain to modify its kinetics and subsequently its processivity (Skiniotis et al., 2004; Lakamper and Meyhofer, 2005). On one hand, cryo-EM studies of the native and digested MTs decorated with minimal motor-domain under different nucleotide conditions revealed that much larger fractions of motor bind to the digested MTs under ADP conditions. Further kinetic studies provided evidence of a previously unknown ADP-state, which is trapped on the digested microtubule (Skiniotis et al., 2004). Consequently, the E-hook has important functions for kinesins ADP and/or binding kinetics. On the other hand, using a different approach with fungal conventional kinesin NcKin, our group provided complementary evidence. We observed reduced processivity and speed on digested microtubules in single molecule fluorescence 
assays, although the neck of NcKin does not carry positive charges. This strongly argues against the proposed anchoring interaction of the neck of conventional kinesin and the E-hook. Together with kinetic experiments, multiple molecule gliding assays and single molecule trapping experiments our results strongly argue for a direct interaction between the Ehook and the motor domain. From the measurement of the stall force we concluded that the removal of the E-hook does not influence strongly bound states (ATP and NT-free) of NcKin, but ADP-states. Binding studies reveal a similar increase in the fraction of $\mathrm{NcKin}$ minimal motors bound to the digested MT, suggesting a common mechanistic function of the E-hook for fungal and animal conventional kinesins (Lakamper and Meyhofer, 2005). The exact mechanism of how the Ehook interacts with the motor remains to be elucidated through further cross-linking and mutational studies. These results stress the complex role of microtubules in kinesin motility and serve as a clear example in which the distinction between NDBS and NIBS reaches its limitations, as the direct influence of structures outside the classical interaction sites on the nucleotide-kinetics becomes evident. Even more interestingly, the tubulin structure which influences kinesin motility most, the E-hook, is the mayor site of differences between tubulin iso-forms (Sullivan and Cleveland, 1986) and subject of extensive post-translational modifications (Westermann and Weber, 2003).

\section{Post-translational modifications on the E-hook of tubulin and their relevance for kinesin motility and cellular function}

Diverse functions of MTs in cell motility and cell architecture are likely to be reflected in tubulin-diversity. As observed for other proteins, the cell uses two mechanisms to provide tubulin variants. Expression of $\alpha \beta$-tubulin iso-types/iso-forms and post-translationally modified tubulin. With the exception of acetylation of $\alpha$-tubulin at the inside of the microtubule, the predominant sources of tubulin-diversity are the mostly disordered C-termini (CTT, E-hooks) on the outer-rim of the microtubule (Wendt et al., 2003; Westermann and Weber, 2003). As described above, these regions coincide with the mayor sites of kinesin interactions with the microtubule. A brief summary of the possible (regulatory) functions of kinesin's post-translational modifications and relations to kinesin is given in Table 1.

While distinct tubulin iso-forms are responsible for very specific functions, like $\gamma$-tubulin in the MTOC, most minor variations in the $\mathrm{C}$-termini of the predominant $\alpha$ - and $\beta$-tubulin monomers (iso-types) seem to be interchangeable (Luduena, 1998). Nevertheless, structurally similar iso-forms may be functionally different (Hutchens et al., 1997). Reports of axonemespecific $\beta$-tubulin motifs and $\beta$-isoform-specific MT-architecture (Raff et al., 1997; Nielsen et al., 2001) were challenged recently by experiments in which $\alpha$ - and $\beta$-CTTs were deleted, interchanged and combined in order to test for the rescue of lethal heterokaryons of $T$. thermophila (Duan and Gorovsky, 2002). Apart form the notion that the tail (CTT) has essential functions not associated with post-translational modifications, this study showed that $T$. thermophila requires sufficiently extended, but interchangeable regions of post-translational modifications on the CTT of at least one tubulin subunit, possibly cross-talking between the $\alpha$ - and $\beta$-subunit through the E-hooks Redeker et al., 2005).

The interpretation that tubulin-distribution in MT and axonemes is reflected in, possibly even caused by, the type of modification in the tail is supported by observations in sea urchin sperm axonemes and Chlamydomonas flagellar axonemes: (1) the A-tubules of

Table 1. Overview over the post-translational tubulin modifications and the proposed relation to kinesin motors

\begin{tabular}{|c|c|c|c|c|}
\hline Modification & Tubulin & Enzymes & Possible function & Proposed relation to kinesin \\
\hline $\begin{array}{l}\text { Tyrosination/ } \\
\text { detyrosination }\end{array}$ & Only $\alpha$ E-hook & TTL & $\begin{array}{l}\text { IF-network } \\
\text { Differentiation (?) }\end{array}$ & Kinesin cross-links IFs to MTs \\
\hline$\Delta 2$-Tubulin & Only $\alpha$ E-hook & Specific TTCP (?) & $\begin{array}{l}\text { Tubulin maturation } \\
\text { Glycylation marker (?) }\end{array}$ & Role of tyrosine in kinesin-motility (?) \\
\hline Glycylation & $\alpha$ and $\beta$ E-hook & $?$ & $\begin{array}{l}\text { Axonemal organization } \\
\text { Cytokinesis }\end{array}$ & $\begin{array}{l}\text { Kinesin-mediated IFT affected (?) } \\
\text { MT-Severing-enzyme transport affected (?) }\end{array}$ \\
\hline Glutamylation & $\alpha$ and $\beta$ E-hook & TTL-like proteins Nek & $\begin{array}{l}\text { Centriole maturation } \\
\text { Axonemal organization } \\
\text { MAP-binding }\end{array}$ & Kinesin-mediated IFT affected (?) \\
\hline & & & & $\begin{array}{l}\text { Interference with kinesin-mediated } \\
\text { transport }\end{array}$ \\
\hline Acetylation & Only $\alpha$ N-Terminal region & HDAC6, SIRT2 & $\begin{array}{l}\text { Cell motility } \\
\text { MAP-binding }\end{array}$ & $\begin{array}{l}\text { Structural effects (?) } \\
\text { Interference with kinesin-mediate } \\
\text { transport }\end{array}$ \\
\hline Palmitoylation & $\alpha, \beta(?)$ & $?$ & $\begin{array}{l}\text { Membrane interactions (?) } \\
\text { Microtubule positioning }\end{array}$ & None so far \\
\hline Phosphorylation & $\beta, \alpha(?)$ & $?$ & Differentiation (?) & None so far \\
\hline
\end{tabular}

TTL, tubulin-tyrosine-ligase; IF, intermediate filament; TTCP, tubulin tyrosin caboxy-peptidase, Nek, NIMA(never in mitosis gene)-related kinase; IFT, intraflagellar transport; MAP, microtubule associated protein; HDAC6, histone deacetylase 6; SIRT2, Sir2 homolog. 
outer axoneme doublets are mostly unmodified (Tyr-tubulin without glytamylation or glycylation) while the B-tubules are glutamylated and detyrosinated (Glu-tubulin, see below) (Multigner et al., 1996; Johnson, 1998; Mencarelli et al., 2000). (2) The axoneme architecture leads to an end-region enriched in tyrosinated, non-modified tubulin. Interestingly, the level of glutamylation and glygcylation decreases towards the end, possibly suggesting modification to be an age marker (Bre et al., 1996; Huitorel et al., 2002). Similarly, while detyrosination does not affect MT-stability, it might serve as an indicator of how long a MT has been assembled (Raybin and Flavin, 1977b; Webster et al., 1990; Gurland and Gundersen, 1995).

Post-translational modifications of the CTT have been described mainly for cells and organisms containing axonemal organelles, but also take place in non-axoneme containing cells. While acetylation of $\alpha$-tubulin on residue 40 is likely to have effects on kinesin motility, this is not discussed in this review as it is not directly linked to the E-hook. The three mayor classes of posttranslational modifications of the E-hook which have implications for kinesin motility and function are tyrosination, and glutamylation and glycylations. Palmitylation and Phosphorylation of tubulin has been observed for $\alpha$ - and $\beta$ - tubulin, respectively, but have not been studied extensively yet.

\section{Tyrosination}

While glycylation and glutamylation occurs in both $\alpha$ - and $\beta$-tubulin, only $\alpha$-tubulin is post-translationally modified by addition or removal of the most $\mathrm{C}$-terminal tyrosine residue (Westermann and Weber, 2003). The C-terminal tyrosine encoded in most isolated mRNAs for $\alpha$-tubulin, is removed by a currently unidentified, maybe non-specific enzyme activity, tubulin tyrosine carboxyl-peptidase, to generate so called Glu-tubulin (TTCP, (Arce et al., 1975; Raybin and Flavin, 1977a,b; Argarana et al., 1978; Argarana et al., 1980). In an ATP-dependent process, mediated by tubulin tyrosine ligase (TTL, (Murofushi, 1980; Ersfeld et al., 1993; Idriss, 2000) can restore the initial C-terminus. Loss of the C-terminal Glutamate of Glu-tubulin yields $\Delta 2$-tubulin, which cannot be tyrosinated by TTL anymore (Paturle-Lafanechere, 1991). It is so far unknown if this deglutamylation is mediated by specific or unspecific proteases. Detyrosination, on the other hand, can be prevented by TTL-mediated incorporation of 3-nitro-tyrosine, which is generated in vivo in response to nitric-oxide (Eiserich et al., 1999; Kalisz et al., 2000). Similarly, TTL catalyzes the irreversible incorporation of Aza-tyrosine into the C-terminus (Purro et al., 2003).

Although the tyrosination cycle of tubulin occurs only at the $\alpha$-tubulin and kinesin binding is predominantly mediated through contacts with the $\beta$-tubulin, influences of this type of modification on the interaction between kinesin and tubulin have been reported.
Kinesin was shown to be necessary for the extended distribution of IFs in the cell, as injection of anti-bodies against $\mathrm{KHC}$ collapsed the IF network to perinuclear locations (Gyoeva and Gelfand, 1991). Furthermore, it could be shown that vimentin-IFs localize preferentially along detyrosinated, Glu-MTs in vivo (Gurland and Gundersen, 1995). Together, these data suggest kinesin to be a preferential cross-linker between vimentin-IFs and Glu-tubulin, not tyr-tubulin. In fact binding studies in vitro and in vivo could confirm that tissue-purified kinesin binds to Glu-MTs with a 2.8-fold higher affinity (Liao and Gundersen, 1998) and that micro-injection of monomeric Glu-tubulin in cells induces the collapse if the extended IF-network most probably due to kinesin depletion from stable Glu-MTs (Kreitzer et al., 1999).

The collapse of the IF network in response to a kinesin depletion has also been observed in a complex chain of events caused by the over-expression of tau in astrocytes (Yoshiyama et al., 2003), high levels of tau led to sharp decrease in stable Glu-MTs accompanied by an increase in tubulin synthesis and accumulation of Tyr-MTs. An observed concomitant decrease of kinesin levels led to the collapse of the IF network and progressive disruption of kinesin-mediated transport. In this study, over-expression of tau ultimately led to atrophy and non-apoptotic cell death. While other studies have linked interference of tau and other structural MAPS with kinesins binding to the MT to the development of Alzheimers (Ebneth et al., 1998; Seitz et al., 2002), this study stresses the role of kinesin and tubulin-tyrosination as a regulatory process (Yoshiyama et al., 2003). Interestingly, several studies reveal that TTL-activity is reduced or absent in breast cancer (Mialhe et al., 2001), and cell-lines with low TTL-activity frequently induce tumor-formation (Lafanechere et al., 1998) due to increased levels of Glu-tubulin. The specific incorporation of Aza-tyrosine could counterbalance of c-Ha-ras mediated tumor induction (Purro et al., 2003). TLL-mediated Nitro-tyrosine incorporation (Kalisz et al., 2000) has been linked to MT dysfunction in lung carcinoma cells (Eiserich et al., 1999) but also to the blocking of early myogenic differentiation L6 Myoblasts (Chang et al., 2002), stressing the possible role of properly tyrosinated MTs in cell maintenance.

There is little structural information available on the cause of the increased affinity of kinesin to Glu-tubulin. The observation that monomeric heads of conventional kinesins bind in ADP-trapped state to MTs without E-hooks (Skiniotis et al., 2004), might provide interesting clues, but little specific information, as the complete E-hook includes other possible sites of post-translational modifications. However, an unexpected increase of ATPase-activity of truncated, monomeric constructs of the fungal conventional kinesin NcKin, NK378 and NK383, on digested microtubules might be connected to tubulin-tyrosination (Lakamper and Meyhofer, 2005), the activity of these constructs on undigested 
tissue-purified MTs is tightly controlled by a highly conserved, inhibitory tyrosine residue (Y362) that is buried in the neck-coiled coil in functional dimeric constructs (Schafer et al., 2003; Bathe et al., 2005). The specific site and mechanism of inhibition is so far unknown, but it is conceivable that the terminal tyrosine of the E-hooks of $\alpha$-tubulin plays a role through unknown mechanisms.

\section{Glycylation}

The reversible addition of 1-40 glycine residue to one or more specific glutamate residue in the flexible E-hook region of both $\alpha$ - and $\beta$-tubulin was first described in Paramecium (Redeker et al., 1994; Rudiger et al., 1995; Mary et al., 1996; Plessmann and Weber, 1997). It is a post-translational mechanism that is unique to tubulin and occurs only in cell types that contain either cilia or flagella and here prominently in the axonemal microtubules (Levilliers et al., 1995; Bre et al., 1998; Xia et al., 2000; Westermann and Weber, 2003). The enzymes catalyzing the addition of the glycines have not been isolated to date.

The possibility to produce heterokaryons in the binucleate ciliate Tetrahymena allowed to study combinations of genetically modified $\alpha$ - and $\beta$-tubulin genes (Redeker et al., 2005). Interestingly, the three-glutamylation sites identified in $\alpha$-tubulin are not essential, whereas similar sites in $\beta$-tubulin are essential, but lethal mutants could be rescued by co-transformation with $\alpha$ tubulin-genes that carried a full length $\beta$-tubulin-E-hook. Unexpectedly, some mutations in the $\beta$-glycylation sites, affected poly-modifications in the $\alpha$-tubulin subunit, suggesting a cross-talk between the two subunits of the tubulin dimer through an unknown mechanism (Redeker et al., 2005). The compromised but viable polyglycylation mutant $\beta_{E D D D D} 440$ grows slow, and has severe effects on the axonemal architecture, most mutants form defective axonemes lacking the central pair of A-tubules. Furthermore, the mutants display reduced motility and are defective in cytokinesis (Xia et al., 2000; Thazhath et al., 2002), possibly suggesting effects on the interaction between the mutated E-hooks with dynein and/or kinesin. However, so far the observed effects have not been convincingly linked to any kinesin, although changes in intra-flagellar transport have been described that could be linked to kinesin-II (Brown, 1999).

\section{Glutamylation}

While polyglycylations have been extensively studied using Tetrahymena heterokaryons, the effect of mutations in the E-hook on polyglutamylation has not yet been studied as extensive using this method. However, polyglutamylation of $\alpha$-tubulin seems to play a key role in flagellar motility (Gagnon et al., 1996; Million et al., 1999). Polygutamylation of multiple neighboring glutamate residues of $\alpha$ - and $\beta$-tubulin is described for many organisms and isoforms (Kann et al., 2003). Very recently the tubulin polyglutamylase could be identified as a TTL-domain enzyme (Janke, 2005). Particularly interesting in the context of kinesin is the observation of prominent polyglutamylation of MTs in neuronal axons, while polyglutamylation in non-neuronal cells is mainly detected in the centrioles, the mitotic spindle and midbody, as well as the primary cilium (Bobinnec et al., 1998).

In contrast to the electrostatically neutral polyglycylation, polyglutamylation adds up to 20 highly negatively charged glutamate-residues to the already negatively charged E-hooks of both $\alpha$ - and $\beta$-tubulin. It could be shown that the affinity of structural MAPs, like tau and MAP2 (Bonnet et al., 2001), as well as kinesin increases progressively by addition of 1-3 glutamate residues, while further glutamate-addition gradually decreases their affinity (Boucher et al., 1994; Larcher et al., 1996). Through the binding of different MAPs to distinct MT-binding sites (similar to the different binding sites of the ncd- tail) it seems to be possible to selectively recruit MAPs depending on the site's ability to be polyglutamylated (Bonnet et al., 2001). An interesting study of the low glutamylation levels of arctic fish tubulin (Redeker et al., 2004) infers very important information about microtubule assembly at low temperatures and might explain the low critical concentrations of subtilisin-treated tubulin (Sackett et al., 1985). However, it also stresses the importance of robust double glutamylation of a specific isotype ( $\beta 4(\mathrm{III}))$ for MAP and motor binding.

\section{Conclusions and outlook}

The literature of tubulin and microtubules contains a significant number of publications that hint at important roles of the structure and/or post-translational modification of $\alpha$-and $\beta$-tubulin. However, a detailed, mechanistic picture of how kinesin's motility and function within the cellular context are modulated is only slowly emerging. One reason for the lack of rapid progress is the difficulty to obtain tubulin-preparations of well-defined post-translational modifications. Therefore, conformational changes within the microtubule might be overlaid or influenced by effects caused by post-translational modifications. The rather crude (and incomplete) approach to remove most sites of posttranslational modification has served well to answer important initial questions, but further understanding is limited. In order to understand the molecular mechanisms that regulate the MT-kinesin interaction it is highly desirable to obtain genetically modified tubulinmutants of defined modification status. Viable Tetrahymena heterokaryons might provide access to more defined tubulin preparations and promise to be helpful in determining if the cell is able to regulate cellular transport and the activity of different kinesins at the level of the microtubule. At the same time these mutants allow to examine the effects on the cellular 
function and viability (Gaertig et al., 1993; 1995). Such investigations should be complemented by detailed biophysical single molecule studies in vitro and in vivo.

\section{Acknowledgements}

We acknowledge financial support from DFG, DARPA, NIH and NSF. Furthermore, we would like to thank Manfred Schliwa and an unknown reviewer for helpful comments on the manuscript.

\section{References}

Alonso MC, Vanderkerckhove J and Cross RA (1998) Proteolytic mapping of kinesin/ncd-microtubule interface: nucleotide-dependent conformational changes in the loops L8 and L12. EMBO J 17: 945-951.

Arce CA, Barra HS, Rodriguez JA and Caputto R (1975) Tentative identification of the amino acid that binds tyrosine as a single unit into a soluble brain protein. FEBS Lett 50: 5-7.

Argarana CE, Barra HS and Caputto R (1978) Release of [14C]tyrosine from tubulinyl-[14C]tyrosine by brain extract. Separation of a carboxypeptidase from tubulin-tyrosine ligase. Mol Cell Biochem 19: $17-21$.

Argarana CE, Barra HS and Caputto R (1980) Tubulinyl-tyrosine carboxypeptidase from chicken brain: properties and partial purification. $J$ Neurochem 34: 114-118.

Asbury CL, Fehr AN and Block SM (2003) Kinesin moves by an asymmetric hand-over-hand mechanism. Science 302: 2130-2134.

Bathe F, Hahlen K, Dombi R, Driller L, Schliwa M and Woehlke G (2005) The complex interplay between the neck and hinge domains in kinesin-1 dimerization and motor activity. Mol Biol Cell 16: 3529-3537.

Bhattacharyya B, Sackett DL and Wolff J (1985) Tubulin, hybrid dimers, and tubulin S. Stepwise charge reduction and polymerization. J Biol Chem 260: 10208-10216.

Bobinnec Y, Moudjou M, Fouquet JP, Desbruyeres E, Edde B and Bornens M (1998) Glutamylation of centriole and cytoplasmic tubulin in proliferating non-neuronal cells. Cell Motil Cytoskeleton 39: $223-232$

Bonnet C, Boucher D, Lazereg S, Pedrotti B, Islam K, Denoulet P and Larcher JC (2001) Differential binding regulation of microtubuleassociated proteins MAP1A, MAP1B, and MAP2 by tubulin polyglutamylation. $J$ Biol Chem 276: 12839-12848.

Boucher D, Larcher JC, Gros F and Denoulet P (1994) Polyglutamylation of tubulin as a progressive regulator of in vitro interactions between the microtubule-associated protein Tau and tubulin. Biochemistry 33: 12471-12477.

Brady ST (1985) A novel brain ATPase with properties expected for the fast axonal transport motor. Nature 317: 73-75.

Bre MH, Redeker V, Quibell M, Darmanaden-Delorme J, Bressac C, Cosson J, Huitorel P, Schmitter JM, Rossler J, Johnson T and others (1996) Axonemal tubulin polyglycylation probed with two monoclonal antibodies: widespread evolutionary distribution, appearance during spermatozoan maturation and possible function in motility. J Cell Sci 109: 727-738.

Bre MH, Redeker V, Vinh J, Rossier J and Levilliers N (1998) Tubulin polyglycylation: differential posttranslational modification of dynamic cytoplasmic and stable axonemal microtubules in paramecium. Mol Biol Cell 9: 2655-2665.

Brown JM, Hardin C and Gaertig J (1999) Rotokinesis, a novel phenomenon of cell locomotion-assisted cytokinesis in the ciliate Tetrahymena thermophila. Cell Biol Int 23: 841-848.

Carter NJ and Cross RA (2005) Mechanics of the kinesin step. Nature 435: $308-312$.
Chang W, Webster DR, Salam AA, Gruber D, Prasad A, Eiserich JP and Bulinski JC (2002) Alteration of the C-terminal amino acid of tubulin specifically inhibits myogenic differentiation. $J$ Biol Chem 277: 30690-30698

Coy DL, Hancock WO, Wagenbach M and Howard J (1999) Kinesin's tail domain is an inhibitory regulator of the motor domain. Nat Cell Biol 1: 288-292.

de Cuevas M, Tao T and Goldstein LS (1992) Evidence that the stalk of Drosophila kinesin heavy chain is an alpha-helical coiled coil. $J$ Cell Biol 116: 957-965.

Duan J and Gorovsky MA (2002) Both carboxy-terminal tails of alpha- and beta-tubulin are essential, but either one will suffice. Curr Biol 12: 313-316.

Ebneth A, Godemann R, Stamer K, Illenberger S, Trinczek B and Mandelkow E (1998) Overexpression of tau protein inhibits kinesin-dependent trafficking of vesicles, mitochondria, and endoplasmic reticulum: implications for Alzheimer's disease. J Cell Biol 143: 777-794

Eiserich JP, Estevez AG, Bamberg TV, Ye YZ, Chumley PH, Beckman JS and Freeman BA (1999) Microtubule dysfunction by posttranslational nitrotyrosination of alpha-tubulin: a nitric oxide-dependent mechanism of cellular injury. Proc Natl Acad Sci USA 96: 6365-6370.

Ersfeld K, Wehland J, Plessmann U, Dodemont H, Gerke V and Weber K (1993) Characterization of the tubulin-tyrosine ligase. $J$ Cell Biol 120: 725-732.

Gaertig J, Cruz MA, Bowen J, Gu L, Pennock DG and Gorovsky MA (1995) Acetylation of lysine 40 in alpha-tubulin is not essential in Tetrahymena thermophila. J Cell Biol 129: 1301-1310.

Gaertig J, Thatcher TH, McGrath KE, Callahan RC and Gorovsky MA (1993) Perspectives on tubulin isotype function and evolution based on the observation that Tetrahymena thermophila microtubules contain a single alpha- and beta-tubulin. Cell Motil Cytoskeleton 25: 243-253.

Gagnon C, White D, Cosson J, Huitorel P, Edde B, Desbruyeres E, Paturle-Lafanechere L, Multigner L, Job D and Cibert C (1996) The polyglutamylated lateral chain of alpha-tubulin plays a key role in flagellar motility. J Cell Sci 109: 1545-1553.

Gilbert SP, Moyer ML and Johnson KA (1998) Alternating site mechanism of the kinesin ATPase. Biochemistry 37: 792-799.

Goldstein LS (1993) With apologies to scheherazade: tails of 1001 kinesin motors. Annu Rev Genet 27: 319-351.

Goldstein LS (2001) Molecular motors: from one motor many tails to one motor many tales. Trends Cell Biol 11: 477-482.

Gurland G and Gundersen GG (1995) Stable, detyrosinated microtubules function to localize vimentin intermediate filaments in fibroblasts. J Cell Biol 131: 1275-1290.

Gyoeva FK and Gelfand VI (1991) Coalignment of vimentin intermediate filaments with microtubules depends on kinesin. Nature 353: 445-448.

Hackney DD (1994) Evidence for alternating head catalysis by kinesin during microtubule-stimulated ATP hydrolysis. Proc Natl Acad Sci USA 91: 6865-6869.

Hirose K, Lockhart A, Cross RA and Amos LA (1995) Nucleotidedependent angular change in kinesin motor domain bound to tubulin. Nature 376: 277-279.

Hirose K, Lowe J, Alonso M, Cross RA and Amos LA (1999) 3D electron microscopy of the interaction of kinesin with tubulin. Cell Struct Funct. 24: 277-284.

Hoenger A and Milligan RA (1997) Motor domains of kinesin and ncd interact with microtubule protofilaments with the same binding geometry. J Mol Biol 265: 553-564.

Hoenger A, Sablin EP, Vale RD, Fletterick RJ and Milligan RA (1995) Three-dimensional structure of a tubulin-motor-protein complex. Nature 376: 271-274.

Howard J, Hudspeth AJ and Vale RD (1989) Movement of microtubules by single kinesin molecules. Nature 342: 154-158.

Huitorel P, White D, Fouquet JP, Kann ML, Cosson J and Gagnon C (2002) Differential distribution of glutamylated tubulin isoforms along the sea urchin sperm axoneme. Mol Reprod Dev 62: 139-148. 
Hutchens JA, Hoyle HD, Turner FR and Raff EC (1997) Structurally similar Drosophila alpha-tubulins are functionally distinct in vivo Mol Biol Cell 8: 481-500.

Idriss HT (2000) Phosphorylation of tubulin tyrosine ligase: a potential mechanism for regulation of alpha-tubulin tyrosination. Cell Motil Cytoskeleton 46: 1-5.

Janke C, Rogowski K, Wloga D, Regnard C, Kajava AV, Strub JM, Temurak N, van Dijk J, Boucher D, van Dorsselaer A and others (2005) Tubulin polyglutamylase enzymes are members of the TTL domain protein family. Science 308: 1758-1762.

Jimenez MA, Evangelio JA, Aranda C, Lopez-Brauet A, Andreu D, Rico M, Lagos R, Andreu JM and Monasterio O (1999) Helicity of alpha(404-451) and beta(394-445) tubulin C-terminal recombinant peptides. Protein Sci 8: 788-799.

Johnson KA (1998) The axonemal microtubules of the Chlamydomonas flagellum differ in tubulin isoform content. J Cell Sci 111: 313-320.

Johnson CS, Buster D and Scholey JM (1990) Light chains of sea urchin kinesin identified by immunoadsorption. Cell Motil Cytoskeleton 16: 204-213.

Kalisz HM, Erck C, Plessmann U and Wehland J (2000) Incorporation of nitrotyrosine into alpha-tubulin by recombinant mammalian tubulin-tyrosine ligase. Biochim Biophys Acta 1481: 131-138.

Kann ML, Soues S, Levilliers N and Fouquet JP (2003) Glutamylated tubulin: diversity of expression and distribution of isoforms. Cell Motil Cytoskeleton 55: 14-25.

Kapoor TM and Compton DA (2002) Searching for the middle ground: mechanisms of chromosome alignment during mitosis. J Cell Biol 157: 551-556.

Karabay A and Walker RA (1999a) Identification of microtubule binding sites in the Ncd tail domain. Biochemistry 38: 1838-1849.

Karabay A and Walker RA (1999b) The Ncd tail domain promotes microtubule assembly and stability. Biochem Biophys Res Commun 258: $39-43$.

Karabay A and Walker RA (2003) Identification of Ncd tail domainbinding sites on the tubulin dimer. Biochem Biophys Res Commun 305: $523-528$.

Kashina AS, Baskin RJ, Cole DG, Wedaman KP, Saxton WM and Scholey JM (1996) A bipolar kinesin. Nature 379: 270-272.

Kirchner J, Seiler S, Fuchs S and Schliwa M (1999) Functional anatomy of the kinesin molecule in vivo. EMBO J 18: 4404-4413.

Klopfenstein DR, Tomishige M, Stuurman N and Vale RD (2002) Role of phosphatidylinositol $(4,5)$ bisphosphate organization in membrane transport by the Unc104 kinesin motor. Cell 109: 347-358.

Krebs A, Goldie KN and Hoenger A (2004) Complex formation with kinesin motor domains affects the structure of microtubules. $J \mathrm{Mol}$ Biol 335: 139-153.

Kreitzer G, Liao G and Gundersen GG (1999) Detyrosination of tubulin regulates the interaction of intermediate filaments with microtubules in vivo via a kinesin-dependent mechanism. Mol Biol Cell 10: 1105-1118.

Kull FJ, Sablin EP, Lau R, Fletterick RJ and Vale RD (1996) Crystal structure of the kinesin motor domain reveals a structural similarity to myosin. Nature 380: $550-555$.

Kuznetsov SA, Vaisberg YA, Rothwell SW, Murphy DB and Gelfand VI (1989) Isolation of a $45-\mathrm{kDa}$ fragment from the kinesin heavy chain with enhanced ATPase and microtubule-binding activities. $J$ Biol Chem 264: 589-595.

Kuznetsov SA, Vaisberg EA, Shanina NA, Magretova NN, Chernyak VY and Gelfand VI (1988) The quaternary structure of bovine brain kinesin. EMBO $J$ 7: 353-356.

Lafanechere L, Courtay-Cahen C, Kawakami T, Jacrot M, Rudiger M, Wehland J, Job D and Margolis RL (1998) Suppression of tubulin tyrosine ligase during tumor growth. $J$ Cell Sci 111: 171-181.

Lakamper S and Meyhofer E (2005) The E-hook of tubulin interacts with kinesin's head to increase processivity and speed. Biophys $J$ 89: 3223-3234.

Lakamper S, Kallipolitou A, Woehlke G, Schliwa M and Meyhofer E (2003) Single fungal kinesin motor molecules move processively along microtubules. Biophys $J$ 84: 1833-1843.
Larcher JC, Boucher D, Lazereg S, Gros F and Denoulet P (1996) Interaction of kinesin motor domains with alpha- and beta-tubulin subunits at a tau-independent binding site. Regulation by polyglutamylation. J Biol Chem 271: 22117-22124.

Lawrence CJ, Dawe RK, Christie KR, Cleveland DW, Dawson SC, Endow SA, Goldstein LS, Goodson HV, Hirokawa N, Howard J and others (2004) A standardized kinesin nomenclature. J Cell Biol 167: 19-22.

Levilliers N, Fleury A and Hill AM (1995) Monoclonal and polyclonal antibodies detect a new type of post-translational modification of axonemal tubulin. J Cell Sci 108: 3013-3028.

Liao G and Gundersen GG (1998) Kinesin is a candidate for crossbridging microtubules and intermediate filaments. Selective binding of kinesin to detyrosinated tubulin and vimentin. $J$ Biol Chem 273: 9797-9803.

Luduena RF (1998) Multiple forms of tubulin: different gene products and covalent modifications. Int Rev Cytol 178: 207-275.

Mary J, Redeker V, Le Caer JP, Rossier J and Schmitter JM (1996) Posttranslational modifications in the C-terminal tail of axonemal tubulin from sea urchin sperm. $J$ Biol Chem 271: 9928-9933.

Mencarelli C, Bre MH, Levilliers N and Dallai R (2000) Accessory tubules and axonemal microtubules of Apis mellifera sperm flagellum differ in their tubulin isoform content. Cell Motil Cytoskeleton 47: 1-12.

Meyhofer E and Howard J (1995) The force generated by a single kinesin molecule against an elastic load. Proc Natl Acad Sci USA 92: $574-578$

Mialhe A, Lafanechere L, Treilleux I, Peloux N, Dumontet C, Bremond A, Panh MH, Payan R, Wehland J, Margolis RL and others (2001) Tubulin detyrosination is a frequent occurrence in breast cancers of poor prognosis. Cancer Res 61: 5024-5027.

Million K, Larcher J, Laoukili J, Bourguignon D, Marano F and Tournier F (1999) Polyglutamylation and polyglycylation of alphaand beta-tubulins during in vitro ciliated cell differentiation of human respiratory epithelial cells. J Cell Sci 112: 4357-4366.

Multigner L, Pignot-Paintrand I, Saoudi Y, Job D, Plessmann U, Rudiger M and Weber K (1996) The A and B tubules of the outer doublets of sea urchin sperm axonemes are composed of different tubulin variants. Biochemistry 35: 10862-10871.

Murofushi H (1980) Purification and characterization of tubulintyrosine ligase from porcine brain. J Biochem (Tokyo) 87: 979-984.

Muto E, Sakai H and Kaseda K (2005) Long-range cooperative binding of kinesin to a microtubule in the presence of ATP. J Cell Biol 168: 691-696.

Nielsen MG, Turner FR, Hutchens JA and Raff EC (2001) Axonemespecific beta-tubulin specialization: a conserved C-terminal motif specifies the central pair. Curr Biol 11: 529-533.

Nitta R, Kikkawa M, Okada Y and Hirokawa N (2004) KIF1A alternately uses two loops to bind microtubules. Science 305: 678-683.

Nogales E, Wolf SG and Downing KH (1998) Structure of the alpha beta tubulin dimer by electron crystallography. Nature 391: 199-203.

Okada Y and Hirokawa N (1999) A processive single-headed motor: kinesin superfamily protein KIF1A. Science 283: 1152-1157.

Okada Y and Hirokawa N (2000) Mechanism of the single-headed processivity: diffusional anchoring between the K-loop of kinesin and the C terminus of tubulin. Proc Natl Acad Sci USA 97: 640-645.

Ovechkina Y, Wagenbach M and Wordeman L (2002) K-loop insertion restores microtubule depolymerizing activity of a "neckless" MCAK mutant. $J$ Cell Biol 159: 557-562.

Paturle-Lafanechere L, Edde B, Denoulet P, Van Dorsselaer A, Mazarguil H, Le Caer JP, Wehland J and Job D (1991) Characterization of a major brain tubulin variant which cannot be tyrosinated. Biochemistry 30: 10523-10528.

Plessmann U and Weber K (1997) Mammalian sperm tubulin: an exceptionally large number of variants based on several posttranslational modifications. J Protein Chem 16: 385-390. 
Purro SA, Bisig CG, Contin MA, Barra HS and Arce CA (2003) Posttranslational incorporation of the antiproliferative agent azatyrosine into the C-terminus of alpha-tubulin. Biochem J 375: 121-129.

Raff EC, Fackenthal JD, Hutchens JA, Hoyle HD and Turner FR (1997) Microtubule architecture specified by a beta-tubulin isoform. Science 275: 70-73.

Raybin D and Flavin M (1977a) Enzyme which specifically add tyrosine to the alpha chain of tubulin. Biochemistry 16: 2189-2194.

Raybin D and Flavin M (1977b) Modification of tubulin by tyrosylation in cells and extracts and its effect on assembly in vitro. J Cell Biol 73: 492-504.

Redeker V, Frankfurter A, Parker SK, Rossier J, Detrich HW and 3rd (2004) Posttranslational modification of brain tubulins from the Antarctic fish Notothenia coriiceps: reduced C-terminal glutamylation correlates with efficient microtubule assembly at low temperature. Biochemistry 43: 12265-12274.

Redeker V, Levilliers N, Schmitter JM, Le Caer JP, Rossier J, Adoutte A and Bre MH (1994) Polyglycylation of tubulin: a posttranslational modification in axonemal microtubules. Science 266: 16881691.

Redeker V, Levilliers N, Vinolo E, Rossier J, Jaillard D, Burnette D, Gaertig J and Bre MH (2005) Mutations of tubulin glycylation sites reveal cross-talk between the $\mathrm{C}$ termini of alpha- and beta-tubulin and affect the ciliary matrix in Tetrahymena. J Biol Chem 280: 596606.

Rice S, Lin AW, Safer D, Hart CL, Naber N, Carragher BO, Cain SM, Pechatnikova E, Wilson-Kubalek EM, Whittaker M and others (1999) A structural change in the kinesin motor protein that drives motility. Nature 402: 778-784.

Rudiger M, Plessmann U, Rudiger AH and Weber K (1995) Beta tubulin of bull sperm is polyglycylated. FEBS Lett 364: 147-151.

Sackett DL, Bhattacharyya B and Wolff J (1985) Tubulin subunit carboxyl termini determine polymerization efficiency. $J$ Biol Chem 260: $43-45$.

Schafer F, Deluca D, Majdic U, Kirchner J, Schliwa M, Moroder L and Woehlke G (2003) A conserved tyrosine in the neck of a fungal kinesin regulates the catalytic motor core. EMBO J 22: 450-458.

Scholey JM, Heuser J, Yang JT and Goldstein LS (1989) Identification of globular mechanochemical heads of kinesin. Nature 338: 355 357.

Seiler S, Kirchner J, Horn C, Kallipolitou A, Woehlke G and Schliwa $M$ (2000) Cargo binding and regulatory sites in the tail of fungal conventional kinesin. Nat Cell Biol 2: 333-338.

Seitz A, Kojima H, Oiwa K, Mandelkow EM, Song YH and Mandelkow E (2002) Single-molecule investigation of the interference between kinesin, tau and MAP2c. EMBO J 21: 4896-4905.

Shiroguchi K, Ohsugi M, Edamatsu M, Yamamoto T and Toyoshima YY (2003) The second microtubule-binding site of monomeric kid enhances the microtubule affinity. $J$ Biol Chem 278: 22460-22465.

Skiniotis G, Cochran JC, Muller J, Mandelkow E, Gilbert SP and Hoenger A (2004) Modulation of kinesin binding by the C-termini of tubulin. EMBO J 23: 989-999.

Song YH and Mandelkow E (1993) Recombinant kinesin motor domain binds to beta-tubulin and decorates microtubules with a B surface lattice. Proc Natl Acad Sci USA 90: 1671-1675.

Song YH, Marx A, Muller J, Woehlke G, Schliwa M, Krebs A, Hoenger A and Mandelkow E (2001) Structure of a fast kinesin: implications for ATPase mechanism and interactions with microtubules. EMBO J 20: 6213-6225.
Stock MF, Chu J and Hackney DD (2003) The kinesin family member BimC contains a second microtubule binding region attached to the $\mathrm{N}$ terminus of the motor domain. $J$ Biol Chem 278: 52315-52322.

Sullivan KF and Cleveland DW (1986) Identification of conserved isotype-defining variable region sequences for four vertebrate beta tubulin polypeptide classes. Proc Natl Acad Sci USA 83: 43274331.

Svoboda K, Schmidt CF, Schnapp BJ and Block SM (1993) Direct observation of kinesin stepping by optical trapping interferometry. Nature 365: 721-727.

Thazhath R, Liu C and Gaertig J (2002) Polyglycylation domain of beta-tubulin maintains axonemal architecture and affects cytokinesis in Tetrahymena. Nat Cell Biol 4: 256-259.

Thorn KS, Ubersax JA and Vale RD (2000) Engineering the processive run length of the kinesin motor. J Cell Biol 151: 1093-1100.

Tucker C and Goldstein LS (1997) Probing the kinesin-microtubule interaction. $J$ Biol Chem 272: 9481-9488.

Vale RD (2003) The molecular motor toolbox for intracellular transport. Cell 112: 467-480.

Vale RD, Coppin CM, Malik F, Kull FJ and Milligan RA (1994) Tubulin GTP hydrolysis influences the structure, mechanical properties, and kinesin-driven transport of microtubules. $J$ Biol Chem 269: 23769-23775.

Vale RD, Funatsu T, Pierce DW, Romberg L, Harada Y and Yanagida T (1996) Direct observation of single kinesin molecules moving along microtubules. Nature 380: 451-453.

Vale RD, Reese TS and Sheetz MP (1985) Identification of a novel force-generating protein, kinesin, involved in microtubule-based motility. Cell 42: 39-50.

Verhey KJ, Lizotte DL, Abramson T, Barenboim L, Schnapp BJ and Rapoport TA (1998) Light chain-dependent regulation of Kinesin's interaction with microtubules. J Cell Biol 143: 1053-1066.

Verhey KJ and Rapoport TA (2001) Kinesin carries the signal. Trends Biochem Sci 26: 545-550.

Wang $Z$ and Sheetz MP (2000) The C-terminus of tubulin increases cytoplasmic dynein and kinesin processivity. Biophys J 78: 1955-1964.

Webster DR, Wehland J, Weber K and Borisy GG (1990) Detyrosination of alpha tubulin does not stabilize microtubules in vivo. J Cell Biol 111: 113-122.

Wendt T, Karabay A, Krebs A, Gross H, Walker R and Hoenger A (2003) A structural analysis of the interaction between ncd tail and tubulin protofilaments. $J$ Mol Biol 333: $541-552$.

Westermann S and Weber K (2003) Post-translational modifications regulate microtubule function. Nat Rev Mol Cell Biol 4: 938-947.

Woehlke G (2001) A look into kinesin's powerhouse. FEBS Lett 508: 291-294.

Woehlke G, Ruby AK, Hart CL, Ly B, Hom-Booher N and Vale RD (1997) Microtubule interaction site of the kinesin motor. Cell 90: 207-216.

Xia L, Hai B, Gao Y, Burnette D, Thazhath R, Duan J, Bre MH, Levilliers N, Gorovsky MA and Gaertig J (2000) Polyglycylation of tubulin is essential and affects cell motility and division in Tetrahymena thermophila. J Cell Biol 149: 1097-1106.

Yildiz A, Tomishige M, Vale RD and Selvin PR (2004) Kinesin walks hand-over-hand. Science 303: 676-678.

Yoshiyama Y, Zhang B, Bruce J, Trojanowski JQ and Lee VM (2003) Reduction of detyrosinated microtubules and Golgi fragmentation are linked to tau-induced degeneration in astrocytes. $J$ Neurosci 23: 10662-10671. 